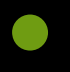 \\ hre \\ 1 \\ a journal of mathematics
}

On the maximum number of isosceles right triangles in a finite point set

Bernardo M. Ábrego, Silvia Fernández-Merchant and David B. Roberts

mathematical sciences publishers 


\title{
On the maximum number of isosceles right triangles in a finite point set
}

\author{
Bernardo M. Ábrego, Silvia Fernández-Merchant and David B. Roberts
}

(Communicated by Kenneth S. Berenhaut)

\begin{abstract}
Let $Q$ be a finite set of points in the plane. For any set $P$ of points in the plane, $S_{Q}(P)$ denotes the number of similar copies of $Q$ contained in $P$. For a fixed $n$, Erdős and Purdy asked for the maximum possible value of $S_{Q}(P)$, denoted by $S_{Q}(n)$, over all sets $P$ of $n$ points in the plane. We consider this problem when $Q=\triangle$ is the set of vertices of an isosceles right triangle. We give exact solutions when $n \leq 9$, and provide new upper and lower bounds for $S_{\triangle}(n)$.
\end{abstract}

\section{Introduction}

Paul Erdős and George Purdy $[1971 ; 1975 ; 1976]$ posed the question: Given a finite set of points $Q$, what is the maximum number $S_{Q}(n)$ of similar copies that can be contained in an n-point set in the plane? This problem remains open in general. However, there has been some progress regarding the order of magnitude of this maximum as a function of $n$. Elekes and Erdôs [1994] noted that $S_{Q}(n) \leq n(n-1)$ for any pattern $Q$ and they also gave a quadratic lower bound for $S_{Q}(n)$ when $|Q|=3$ or when all the coordinates of the points in $Q$ are algebraic numbers. They also proved a slightly subquadratic lower bound for all other patterns $Q$. Later, Laczkovich and Ruzsa [1997] characterized precisely those patterns $Q$ for which $S_{Q}(n)=\Theta\left(n^{2}\right)$. In spite of this, the coefficient of the quadratic term is not known for any nontrivial pattern; it is not even known if $\lim _{n \rightarrow \infty} S_{Q}(n) / n^{2}$ exists!

Apart from being a natural question in discrete geometry, this problem also arose in connection with the optimization of algorithms designed to look for patterns among data obtained from scanners, digital cameras, telescopes, and so on [Brass 2002; Brass et al. 2005; Brass and Pach 2005].

Our paper considers the case where $Q$ is the set of vertices of an isosceles right triangle. The case where $Q$ is the set of vertices of an equilateral triangle has been considered in [Ábrego and Fernández-Merchant 2000]. To avoid redundancy, we

MSC2000: primary 52C10; secondary 05C 35 .

Keywords: Erdős problems, similar triangles, isosceles right triangles.

Supported in part by CURM, BYU, and the NSF (DMS-0636648). 
refer to an isosceles right triangle as an IRT for the remainder of the paper. We begin with some definitions. Let $P$ denote a finite set of points in the plane. We define $S_{\triangle}(P)$ to be the number of triplets in $P$ that are the vertices of an IRT. Furthermore, let

$$
S_{\triangle}(n)=\max _{|P|=n} S_{\triangle}(P) .
$$

As mentioned before, Elekes and Erdôs established that $S_{\triangle}(n)=\Theta\left(n^{2}\right)$ and it is implicit from their work that $1 / 18 \leq \liminf _{n \rightarrow \infty} S_{\triangle}(n) / n^{2} \leq 1$. The main goal of this paper is to derive improved constants that bound the function $S_{\triangle}(n) / n^{2}$. Specifically, in Sections 2 and 3, we prove:

Theorem 1. $\quad 0.433064<\liminf _{n \rightarrow \infty} \frac{S_{\triangle}(n)}{n^{2}} \leq \frac{2}{3}<0.66667$.

We proceed to determine in Section 4 the exact values of $S_{\triangle}(n)$ when $3 \leq n \leq 9$. Several ideas for the proofs of these bounds come from the equivalent bounds for equilateral triangles in [Ábrego and Fernández-Merchant 2000].

\section{Lower bound}

For $z \in P$, let $R_{\pi / 2}(z, P)$ be the $\pi / 2$ counterclockwise rotation of $P$ with center $z$. Let $\operatorname{deg}_{\pi / 2}(z)$ be the number of isosceles right triangles in $P$ such that $z$ is the right-angle vertex of the triangle. If $z \in P$, then $\operatorname{deg}_{\pi / 2}(z)$ can be computed by simply rotating our point set $P$ by $\pi / 2$ about $z$ and counting the number of points in the intersection other than $z$. Therefore,

$$
\operatorname{deg}_{\pi / 2}(z)=\left|P \cap R_{\pi / 2}(z, P)\right|-1 .
$$

Since an IRT has only one right angle,

$$
S_{\triangle}(P)=\sum_{z \in P} \operatorname{deg}_{\pi / 2}(z) .
$$

That is, the sum computes the number of IRTs in $P$. From this identity an initial lower bound of $\frac{5}{12}$ can be derived for $\liminf _{n \rightarrow \infty} S_{\triangle}(n) / n^{2}$ using the set

$$
P=\left\{(x, y) \in \mathbb{Z}^{2}: 0 \leq x \leq \sqrt{n}, 0 \leq y \leq \sqrt{n}\right\} .
$$

We now improve this bound.

The following theorem generalizes our method for finding a lower bound. We denote by $\Lambda$ the lattice generated by the points $(1,0)$ and $(0,1)$, and we refer to points in $\Lambda$ as lattice points. The next result provides a formula for the leading term of $S_{\triangle}(P)$ when our points in $P$ are lattice points enclosed by a given shape. This theorem, its proof, and notation, are similar to those of Theorem 2 in [Ábrego and Fernández-Merchant 2000], where we obtained a similar result for equilateral triangles in place of IRTs. 
Theorem 2. Let $K$ be a compact set with finite perimeter and area 1 . Define

$f_{K}: \mathbb{C} \rightarrow \mathbb{R}^{+} \quad$ as $f_{K}(z)=\operatorname{Area}\left(K \cap R_{\pi / 2}(z, K)\right), \quad$ where $z \in K$.

If $K_{n}$ is a similar copy of $K$ intersecting $\Lambda$ in exactly $n$ points, then

$$
S_{\triangle}\left(K_{n} \cap \Lambda\right)=\left(\int_{K} f_{K}(z) d z\right) n^{2}+O\left(n^{3 / 2}\right) .
$$

Proof. Given a compact set $L$ with finite area and perimeter, we have

$$
|r L \cap \Lambda|=\operatorname{Area}(r L)+O(r)=r^{2} \operatorname{Area}(L)+O(r),
$$

where $r L$ is the scaling of $L$ by a factor $r$. Therefore,

$$
\begin{aligned}
S_{\triangle}\left(K_{n} \cap \Lambda\right) & =\sum_{z \in K_{n} \cap \Lambda}\left|\left(\Lambda \cap K_{n}\right) \cap R_{\pi / 2}\left(z,\left(K_{n} \cap \Lambda\right)\right)\right|-1 \\
& =\sum_{z \in K_{n} \cap \Lambda} \operatorname{Area}\left(K_{n} \cap R_{\pi / 2}\left(z, K_{n}\right)\right)+O(\sqrt{n}) .
\end{aligned}
$$

We see that each error term in the sum is bounded by the perimeter of $K_{n}$, which is finite by hypothesis. Thus,

$$
\begin{aligned}
S_{\triangle}\left(K_{n} \cap \Lambda\right) & =n^{2} \sum_{z \in K_{n} \cap \Lambda} \frac{1}{n^{2}} \operatorname{Area}\left(K_{n} \cap R_{\pi / 2}\left(z, K_{n}\right)\right)+O\left(n^{3 / 2}\right) \\
& =n^{2} \sum_{z \in K_{n} \cap \Lambda} \frac{1}{n} \operatorname{Area}\left(\frac{1}{\sqrt{n}}\left(K_{n} \cap R_{\pi / 2}\left(z, K_{n}\right)\right)\right)+O\left(n^{3 / 2}\right) \\
& =n^{2} \sum_{z \in K_{n} \cap \Lambda} \frac{1}{n} \operatorname{Area}\left(\frac{1}{\sqrt{n}} K_{n} \cap R_{\pi / 2}\left(\frac{z}{\sqrt{n}}, \frac{1}{\sqrt{n}} K_{n}\right)\right)+O\left(n^{3 / 2}\right) .
\end{aligned}
$$

The last sum is a Riemann approximation for the function $f_{(1 / \sqrt{n}) K_{n}}$ over the region $(1 / \sqrt{n}) K_{n}$; thus

$$
S_{\triangle}\left(K_{n} \cap \Lambda\right)=n^{2}\left(\int_{\frac{1}{\sqrt{n}} K_{n}} f_{\frac{1}{\sqrt{n}} K_{n}}(z) d z+O\left(\frac{1}{\sqrt{n}}\right)\right)+O\left(n^{3 / 2}\right) .
$$

Since

$$
\begin{aligned}
\operatorname{Area}\left(\frac{1}{\sqrt{n}} K_{n}\right) & =\frac{1}{n} \operatorname{Area}\left(K_{n}\right)=\frac{1}{n}(n+O(\sqrt{n})) \\
& =1+O\left(\frac{1}{\sqrt{n}}\right)=\operatorname{Area}(K)+O\left(\frac{1}{\sqrt{n}}\right),
\end{aligned}
$$

it follows that

$$
\int_{\frac{1}{\sqrt{n}} K_{n}} f_{\frac{1}{\sqrt{n}} K_{n}}(z) d z=\int_{K} f_{K}(z) d z+O\left(\frac{1}{\sqrt{n}}\right) .
$$


As a result,

$$
S_{\triangle}\left(K_{n} \cap \Lambda\right)=n^{2} \int_{\frac{1}{\sqrt{n}} K_{n}} f_{\frac{1}{\sqrt{n}} K_{n}}(z) d z+O\left(n^{3 / 2}\right)=n^{2} \int_{K} f_{K}(z) d z+O\left(n^{3 / 2}\right) .
$$

The importance of this theorem can be seen immediately. Although our lower bound of $\frac{5}{12}$ for $\liminf _{n \rightarrow \infty} S_{\triangle}(n) / n^{2}$ was derived by summing the degrees of each point in a square lattice, the same result can be obtained by letting $K$ be the square $\left\{(x, y):|x| \leq \frac{1}{2},|y| \leq \frac{1}{2}\right\}$. It follows that $f_{K}(x, y)=(1-|x|-|y|)(1-|| x|-| y||)$ and

$$
S_{\triangle}\left(K_{n} \cap \Lambda\right)=\left(\int_{K} f_{K}(z) d z\right) n^{2}+O\left(n^{3 / 2}\right)=\frac{5}{12} n^{2}+O\left(n^{3 / 2}\right) .
$$

An improved lower bound will follow provided that we find a set $K$ such that the value for the integral in Theorem 2 is larger than $\frac{5}{12}$. We get a larger value for the integral by letting $K$ be the circle $\{z \in \mathbb{C}:|z| \leq 1 / \sqrt{\pi}\}$. In this case

$$
f_{K}(z)=\frac{2}{\pi} \arccos \left(\frac{\sqrt{2 \pi}}{2}|z|\right)-|z| \sqrt{\frac{2}{\pi}-|z|^{2}}
$$

and

$$
S_{\triangle}\left(K_{n} \cap \Lambda\right)=\left(\int_{K} f_{K}(z) d z\right) n^{2}+O\left(n^{3 / 2}\right)=\left(\frac{3}{4}-\frac{1}{\pi}\right) n^{2}+O\left(n^{3 / 2}\right) .
$$

It was conjectured in [Ábrego and Fernández-Merchant 2000] that not only does $\lim _{n \rightarrow \infty} E(n) / n^{2}$ exist, but it is attained by the uniform lattice in the shape of a circle. $(E(n)$ denotes the maximum number of equilateral triangles determined by $n$ points in the plane.) The corresponding conjecture in the case of the isosceles right triangle turns out to be false. That is, if $\lim _{n \rightarrow \infty} S_{\triangle}(n) / n^{2}$ exists, then it must be strictly greater than $\frac{3}{4}-\pi^{-1}$. Define $\bar{\Lambda}$ to be the translation of $\Lambda$ by the vector $\left(\frac{1}{2}, \frac{1}{2}\right)$. The following lemma will help us to improve our lower bound.

Lemma 3. If $(j, k) \in \mathbb{R}^{2}$ and $\Lambda^{\prime}=\Lambda$ or $\Lambda^{\prime}=\bar{\Lambda}$, then

$$
R_{\pi / 2}\left((j, k), \Lambda^{\prime}\right) \cap \Lambda^{\prime}= \begin{cases}\Lambda^{\prime} & \text { if }(j, k) \in \Lambda \cup \bar{\Lambda}, \\ \varnothing & \text { else. }\end{cases}
$$

Proof. Observe that

$$
R_{\pi / 2}((j, k),(s, t))=\left(\begin{array}{rr}
0 & -1 \\
1 & 0
\end{array}\right)\left(\begin{array}{l}
s-j \\
t-k
\end{array}\right)+\left(\begin{array}{l}
j \\
k
\end{array}\right)=\left(\begin{array}{l}
k-t+j \\
s-j+k
\end{array}\right) .
$$

First suppose $(s, t) \in \Lambda$. Since $s, t \in \mathbb{Z}$, then $(k-t+j, s-j+k) \in \Lambda$ if and only if $k-j \in \mathbb{Z}$ and $k+j \in \mathbb{Z}$. This can only happen when either both $j$ and $k$ are half-integers (i.e., $(j, k) \in \bar{\Lambda}$ ), or both $j$ and $k$ are integers (i.e., $(j, k) \in \Lambda$ ). Now suppose $(s, t) \in \bar{\Lambda}$. In this case, because both $s$ and $t$ are half-integers, we conclude 
that $(k-t+j, s-j+k) \in \bar{\Lambda}$ if and only if both $k-j \in \mathbb{Z}$ and $k+j \in \mathbb{Z}$. Once again this occurs if and only if $(j, k) \in \Lambda \cup \bar{\Lambda}$.

Recall that if $K$ denotes the circle of area 1 , then $\left(\frac{3}{4}-\pi^{-1}\right) n^{2}$ is the leading term of $S_{\triangle}\left(K_{n} \cap \Lambda\right)$. The previous lemma implies that, if we were to adjoin a point $z \in \mathbb{R}^{2}$ to $K_{n} \cap \Lambda$ such that $z$ has half-integer coordinates and is located near the center of the circle formed by the points of $K_{n} \cap \Lambda$, then $\operatorname{deg}_{\pi / 2}(z)$ will approximately equal $\left|K_{n} \cap \Lambda\right|$. We obtain the next theorem by further exploiting this idea.

Theorem 4.

$$
0.43169 \approx \frac{3}{4}-\frac{1}{\pi}<0.433064<\liminf _{n \rightarrow \infty} \frac{S_{\triangle}(n)}{n^{2}} .
$$

Proof. Let $K$ be the circle of area 1 and set $A=K_{m_{1}} \cap \Lambda, B=K_{m_{2}} \cap \bar{\Lambda}$. Position $B$ so that its points are centered on the circle formed by the points in $A$ (see Figure 1). We let $n=m_{1}+m_{2}=|A \cup B|$ and $m_{2}=x \cdot m_{1}$, where $0<x<1$ is a constant to be determined.

We proceed to maximize the leading coefficient of $S_{\triangle}(A \cup B)$ as $x$ varies from 0 to 1 . By Lemma 3, there cannot exist an IRT whose right-angle vertex lies in $A$ while one $\pi / 4$ vertex lies in $A$ and the other lies in $B$. Similarly, there cannot exist an IRT whose right angle-vertex lies in $B$ while one $\pi / 4$ vertex lies in $A$ and the other lies in $B$. Therefore, each IRT with vertices in $A \cup B$ must fall under one of four cases:

Case 1: All three vertices in A. By Theorem 2, there are $\left(\frac{3}{4}-\pi^{-1}\right) m_{1}^{2}+O\left(m_{1}^{3 / 2}\right)$ IRTs in this case. Since $m_{1}=n /(1+x)$, the number of IRTs in terms of $n$ equals

$$
\left(\frac{3}{4}-\frac{1}{\pi}\right) \frac{n^{2}}{(1+x)^{2}}+O\left(n^{3 / 2}\right) .
$$
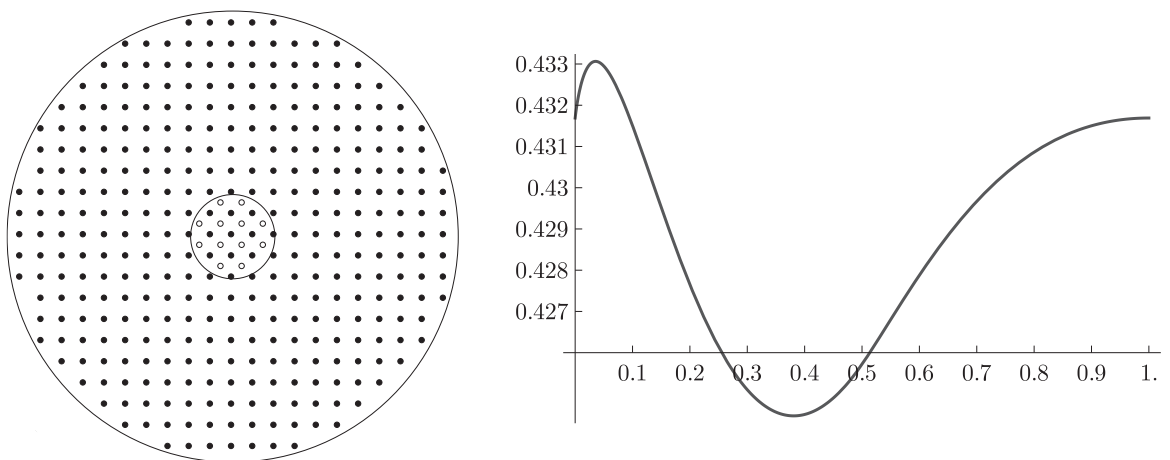

Figure 1. Left: set $B$ (open dots) centered on set $A$ (black dots). Right: plot of the $n^{2}$ coefficient of $S_{\triangle}(A \cup B)$ for $x$ from 0 to 1 . 
Case 2: All three vertices in B. By Theorem 2, there are $\left(\frac{3}{4}-\pi^{-1}\right) m_{2}^{2}+O\left(m_{2}^{3 / 2}\right)$ IRTs in this case. This time $m_{2}=n x /(1+x)$ and the number of IRTs in terms of $n$ equals

$$
\left(\frac{3}{4}-\frac{1}{\pi}\right) \frac{n^{2} x^{2}}{(1+x)^{2}}+O\left(n^{3 / 2}\right)
$$

Case 3: Right-angle vertex in $B, \pi / 4$ vertices in $A$. The relationship given by Lemma 3 allows us to slightly adapt the proof of Theorem 2 in order to compute the number of IRTs in this case. The integral approximation to the number of IRTs in this case is given by

$$
\sum_{z \in K_{m_{2}} \cap \bar{\Lambda}}\left|\left(K_{m_{1}} \cap \Lambda\right) \cap R_{\pi / 2}\left(z,\left(K_{m_{1}} \cap \Lambda\right)\right)\right|=m_{1}^{2}\left(\int_{\frac{1}{\sqrt{m_{1}}} K_{m_{2}}} f_{\frac{1}{\sqrt{m_{1}}} K_{m_{1}}}(z) d z\right)+O\left(m_{1}^{3 / 2}\right) .
$$

But

$$
\operatorname{Area}\left(\frac{1}{\sqrt{m_{1}}} K_{m_{2}}\right)=\operatorname{Area}\left(\sqrt{\frac{m_{2}}{m_{1}}} K\right)+O\left(\sqrt{m_{1}}\right)
$$

so

$$
m_{1}^{2}\left(\int_{\frac{1}{\sqrt{m_{1}}} K_{m_{2}}} f_{\frac{1}{\sqrt{m_{1}}} K_{m_{1}}}(z) d z\right)+O\left(m_{1}^{3 / 2}\right)=m_{1}^{2}\left(\int_{\sqrt{\frac{m_{2}}{m_{1}}} K} f_{K}(z) d z\right)+O\left(m_{1}^{3 / 2}\right) .
$$

Expressing this value in terms of $n$ gives

$$
\left(\int_{\sqrt{x} K} f_{K}(z) d z\right) \frac{n^{2}}{(1+x)^{2}}+O\left(n^{3 / 2}\right) .
$$

Case 4: Right-angle vertex in A, $\pi / 4$ vertices in $B$. As in Case 3, the number of IRTs is given by

$$
\begin{aligned}
\sum_{z \in K_{m_{1}} \cap \Lambda}\left|\left(K_{m_{2}} \cap \bar{\Lambda}\right) \cap R_{\pi / 2}\left(z,\left(K_{m_{2}} \cap \bar{\Lambda}\right)\right)\right| \\
=m_{2}^{2}\left(\int_{\frac{1}{\sqrt{m_{2}}} K_{m_{1}}} f_{\frac{1}{\sqrt{m_{2}}} K_{m_{2}}}(z) d z\right)+O\left(m_{2}^{3 / 2}\right) .
\end{aligned}
$$

Now recall that $f_{\left(1 / \sqrt{m_{2}}\right) K_{m_{2}}}(z)=$ Area $\left(\left(1 / \sqrt{m_{2}}\right) K_{m_{2}} \cap R_{\pi / 2}\left(z,\left(1 / \sqrt{m_{2}}\right) K_{m_{2}}\right)\right)$. It follows that $f_{\left(1 / \sqrt{m_{2}}\right) K_{m_{2}}}\left(z_{0}\right)=0$ if and only if $z_{0}$ is farther than $\sqrt{2 / \pi}$ from the center of $\left(1 / \sqrt{m_{2}}\right) K_{m_{2}}$. Thus for small enough values of $m_{2}$, the region of integration in (6) is actually $\left(\sqrt{2 / m_{2}}\right) K_{m_{2}}$, so it does not depend on $m_{1}$. We consider two subcases.

First, if $x \leq \frac{1}{2}$ (i.e., $m_{2} \leq m_{1} / 2$ ), then

$$
\sqrt{\frac{2}{\pi}}=\frac{1}{\sqrt{m_{2}}} \frac{\sqrt{2 m_{2}}}{\sqrt{\pi}} \leq \frac{1}{\sqrt{m_{2}}} \frac{\sqrt{2}}{\sqrt{\pi}} \frac{\sqrt{m_{1}}}{\sqrt{2}}=\frac{1}{\sqrt{m_{2}}} \sqrt{\frac{m_{1}}{\pi}} .
$$


The left side of this inequality is the radius of $\left(\sqrt{2 / m_{2}}\right) K_{m_{2}}$, while the right side is the radius of $\left(1 / \sqrt{m_{2}}\right) K_{m_{1}}$; thus the region of integration where $f_{\left(1 / \sqrt{m_{2}}\right) K_{m_{2}}}$ is nonzero equals $\left(\sqrt{2 / m_{2}}\right) K_{m_{2}}$. Hence, the number of IRTs equals

$$
\begin{aligned}
m_{2}^{2}\left(\int_{\sqrt{\frac{2}{m_{2}}} K_{m_{2}}} f_{\frac{1}{\sqrt{m_{2}}} K_{m_{2}}}(z) d z\right)+O\left(m_{2}^{3 / 2}\right) & =m_{2}^{2}\left(\int_{\sqrt{2} K} f_{K}(z) d z\right)+O\left(m_{2}^{3 / 2}\right) \\
& =\left(\int_{\sqrt{2} K} f_{K}(z) d z\right) \frac{n^{2} x^{2}}{(1+x)^{2}}+O\left(n^{3 / 2}\right) .
\end{aligned}
$$

Now we consider the case $x>\frac{1}{2}$ (i.e., $m_{2}>m_{1} / 2$ ). In this case, $f_{\left(1 / \sqrt{m_{2}}\right) K_{m_{2}}}$ is nonzero for all points in $\left(1 / \sqrt{m_{2}}\right) K_{m_{1}}$. Thus the number of IRTs is then

$$
\begin{aligned}
m_{2}^{2}\left(\int_{\frac{1}{\sqrt{m_{2}}} K_{m_{1}}} f_{\frac{1}{\sqrt{m_{2}}} K_{m_{2}}}(z) d z\right)+O\left(m_{2}^{3 / 2}\right) & =m_{2}^{2}\left(\int_{\sqrt{\frac{m_{1}}{m_{2}}} K} f_{K}(z) d z\right)+O\left(m_{2}^{3 / 2}\right) \\
& =\left(\int_{\sqrt{\frac{1}{x}} K} f_{K}(z) d z\right) \frac{n^{2} x^{2}}{(1+x)^{2}}+O\left(n^{3 / 2}\right) .
\end{aligned}
$$

By (2), we have, for $t>0$,

$$
\begin{aligned}
\int_{t K} f_{K}(z) d z & =2 \pi \int_{0}^{t / \sqrt{\pi}}\left(\frac{2}{\pi} \arccos \left(\frac{\sqrt{2 \pi}}{2} r\right)-r \sqrt{\frac{2}{\pi}-r^{2}}\right) r d r \\
& =\frac{1}{2 \pi}\left(4 t^{2} \arccos \frac{t}{\sqrt{2}}+2 \arcsin \frac{t}{\sqrt{2}}-t\left(t^{2}+1\right) \sqrt{2-t^{2}}\right) .
\end{aligned}
$$

Therefore, putting all four cases together - i.e., expressions (3)-(5), and either (7) or (8) - we obtain that the $n^{2}$ coefficient of $S_{\triangle}(A \cup B)$ equals

$$
\begin{aligned}
\frac{1}{4 \pi(x+1)^{2}}\left(8 x \arccos \sqrt{\frac{x}{2}}+4 \arcsin \sqrt{\frac{x}{2}}+(5 \pi-4) x^{2}+\right. & (3 \pi-4) \\
& \left.-2(x+1) \sqrt{2 x-x^{2}}\right)
\end{aligned}
$$

if $0<x \leq \frac{1}{2}$, or

$$
\begin{array}{r}
\frac{1}{4 \pi(x+1)^{2}}\left(8 x\left(\arccos \sqrt{\frac{x}{2}}+\arccos \sqrt{\frac{1}{2 x}}\right)+4 \arcsin \sqrt{\frac{x}{2}}+4 x^{2} \arcsin \sqrt{\frac{1}{2 x}}\right. \\
\left.+(3 \pi-4)\left(x^{2}+1\right)-2(x+1)\left(\sqrt{2 x-x^{2}}+\sqrt{2 x-1}\right)\right),
\end{array}
$$

if $\frac{1}{2}<x<1$. Letting $x$ vary from 0 to 1 , this coefficient is maximized (see Figure 1 ) when $x \approx 0.0356067$, corresponding to a radius of $B$ approximately $18.87 \%$ of the radius of $A$. Letting $x$ equal this value gives 0.433064 as a decimal approximation to the maximum value attained by the $n^{2}$ coefficient. 
At this point, one might be tempted to further increase the quadratic coefficient by placing a third set of lattice points arranged in a circle and centered on the circle formed by $B$. It turns out that forming such a configuration does not improve the results in the previous theorem. This is due to Lemma 3. More specifically, given our construction from the previous theorem, there is no place to adjoin a point $z$ to the center of $A \cup B$ such that $z \in \Lambda$ or $z \in \bar{\Lambda}$. Hence, if we were to add the point $z$ to the center of $A \cup B$, then any new IRTs would have their right-angle vertex located at $z$ with one $\pi / 4$ vertex in $A$ and the other $\pi / 4$ vertex in $B$. Doing so can produce at most $2 m_{2}=2 x m_{1} \approx 0.0712 m_{1}$ new IRTs (recall that $x \approx 0.0356066$ in our construction). On the other hand, adding $z$ to the perimeter of $A$, gives us $m_{1} f_{K}(1 / \sqrt{\pi}) \approx 0.1817 m_{1}$ new IRTs.

\section{Upper bound}

We now turn our attention to finding an upper bound for $S_{\triangle}(n) / n^{2}$. It is easy to see that $S_{\triangle}(n) \leq n^{2}-n$, since any pair of points can be the vertices of at most six IRTs. Our next theorem improves this bound. The idea is to prove that there exists a point in $P$ that does not belong to many IRTs. First, we need the following definition.

For every $z \in P$, let $R_{\pi / 4}^{+}(z, P)$ and $R_{\pi / 4}^{-}(z, P)$ be the dilations of $P$, centered at $z$, with factor $\sqrt{2}$ and $1 / \sqrt{2}$, respectively, followed by a $\pi / 4$ counterclockwise rotation with center $z$. Let $\operatorname{deg}_{\pi / 4}^{+}(z)$ and $\operatorname{deg}_{\pi / 4}^{-}(z)$ be the number of isosceles right triangles $z x y$ with $x, y \in P$ such that $z x y$ occur in counterclockwise order, and $z y$, respectively $z x$, is the hypotenuse of the triangle $z x y$.

Much like the case of $\operatorname{deg}_{\pi / 2}, \operatorname{deg}_{\pi / 4}^{+}$and $\operatorname{deg}_{\pi / 4}^{-}$can be computed with the identities

$$
\operatorname{deg}_{\pi / 4}^{+}(z)=\left|P \cap R_{\pi / 4}^{+}(z, P)\right|-1, \quad \operatorname{deg}_{\pi / 4}^{-}(z)=\left|P \cap R_{\pi / 4}^{-}(z, P)\right|-1 .
$$

Theorem 5. $\quad S_{\triangle}(n) \leq\left\lfloor\frac{2}{3}(n-1)^{2}-\frac{5}{3}\right\rfloor$ for $n \geq 3$.

Proof. By induction on $n$. If $n=3$, then $S_{\triangle}(3) \leq 1=\left\lfloor\frac{1}{3}(2 \cdot 4-5)\right\rfloor$. Now suppose the theorem holds for $n=k$. We must show this implies the theorem holds for $n=k+1$. Suppose that there is a point $z \in P$ such that $\operatorname{deg}_{\pi / 2}(z)+\operatorname{deg}_{\pi / 4}^{+}(z)+\operatorname{deg}_{\pi / 4}^{-}(z) \leq$ $\left\lfloor\frac{1}{3}(4 n-5)\right\rfloor$. Then, by induction,

$$
\begin{aligned}
S_{\triangle}(k+1) & \leq \operatorname{deg}_{\pi / 2}(z)+\operatorname{deg}_{\pi / 4}^{+}(z)+\operatorname{deg}_{\pi / 4}^{-}(z)+S_{\triangle}(k) \\
& \leq\left\lfloor\frac{1}{3}(4 k-1)\right\rfloor+\left\lfloor\frac{2}{3}(k-1)^{2}-\frac{5}{3}\right\rfloor=\left\lfloor\frac{2}{3} k^{2}-\frac{5}{3}\right\rfloor .
\end{aligned}
$$

The last equality can be verified by considering the three possible residues of $k$ when divided by 3 . Hence, our theorem is proved if we can find a point $z \in P$ with the desired property. 
Let $x, y \in P$ be points such that $x$ and $y$ form the diameter of $P$. In other words, if $w \in P$, then the distance from $w$ to any other point in $P$ is less than or equal to the distance from $x$ to $y$. We now prove that either $x$ or $y$ is a point with the desired property mentioned above. We begin by analyzing $\operatorname{deg}_{\pi / 4}^{-}$. We use the notation from [Ábrego and Fernández-Merchant 2000, Theorem 1].

Define $N_{x}=P \cap R_{\pi / 4}^{-}(x, P) \backslash\{x\}$ and $N_{y}=P \cap R_{\pi / 4}^{-}(y, P) \backslash\{y\}$. It follows from our identities that, $\operatorname{deg}_{\pi / 4}^{-}(x)=\left|N_{x}\right|$ and $\operatorname{deg}_{\pi / 4}^{-}(y)=\left|N_{y}\right|$. Furthermore, by the inclusion-exclusion principle for finite sets, we have

$$
\left|N_{x}\right|+\left|N_{y}\right|=\left|N_{x} \cup N_{y}\right|+\left|N_{x} \cap N_{y}\right| .
$$

We shall prove by contradiction that $\left|N_{x} \cap N_{y}\right| \leq 1$. Suppose that there are two points $u, v \in N_{x} \cap N_{y}$. This means that there are points $u_{x}, v_{x}, u_{y}, v_{y} \in P$ such that the triangles $x u_{x} u, x v_{x} v, y u_{y} u, y v_{y} v$ are IRTs oriented counterclockwise with right angle at either $u$ or $v$.

But notice that the line segments $u_{x} u_{y}$ and $v_{x} v_{y}$ are simply the $(\pi / 2)$-counterclockwise rotations of $x y$ about centers $u$ and $v$, respectively. Hence, $u_{x} u_{y} v_{x} v_{y}$ is a parallelogram with two sides having length $x y$ as shown in Figure 2, left. This is a contradiction since one of the diagonals of the parallelogram is longer than any of its sides. Thus, $\left|N_{x} \cap N_{y}\right| \leq 1$. Furthermore, $x \notin N_{y}$ and $y \notin N_{x}$, so $\left|N_{x} \cup N_{y}\right| \leq n-2$ and thus $\operatorname{deg}_{\pi / 4}^{-}(x)+\operatorname{deg}_{\pi / 4}^{-}(y)=\left|N_{x} \cup N_{y}\right|+\left|N_{x} \cap N_{y}\right| \leq n-2+1=n-1$. This also implies that $\operatorname{deg}_{\pi / 4}^{+}(x)+\operatorname{deg}_{\pi / 4}^{+}(y) \leq n-1$, since we can follow the same argument applied to the reflection of $P$ about the line $x y$.

We now look at $\operatorname{deg}_{\pi / 2}(x)$ and $\operatorname{deg}_{\pi / 2}(y)$. We claim that, for every $p \in P$, at most one of $R_{\pi / 2}(x, p)$ or $R_{\pi / 2}(y, p)$ belongs to $P$. Indeed, let $p_{x}=R_{\pi / 2}(x, p)$ and $p_{y}=R_{\pi / 2}(y, p)$ (see Figure 2, right). The distance $p_{x} p_{y}$ is exactly the distance $x y$ but scaled by $\sqrt{2}$. This contradicts the fact that $x y$ is the diameter of $P$.

Define a graph $G$ with vertex set $V(G)=P \backslash\{x, y\}$ and edge set given by saying that $u v \in E(G)$ if and only if $v=R_{\pi / 2}(x, u)$ or $v=R_{\pi / 2}(y, u)$. We show that

$$
0 \leq \operatorname{deg}_{\pi / 2}(x)+\operatorname{deg}_{\pi / 2}(y)-|E(G)| \leq 1 \text {. }
$$
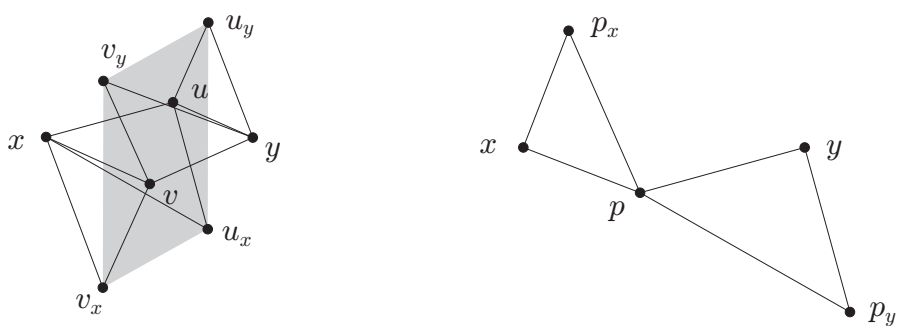

Figure 2. Proof of Theorem 5. 
The left inequality follows from the fact each edge counts an IRT in either $\operatorname{deg}_{\pi / 2}(x)$ or $\operatorname{deg}_{\pi / 2}(y)$ and possibly in both. However, if $u v$ is an edge of $G$ so that $v=R_{\pi / 2}(x, u)$ and $u=R_{\pi / 2}(y, v)$, then $x u y v$ is a square, so this can only happen for at most one edge.

Now, let $\operatorname{deg}_{G}(u)$ be the number of edges in $E(G)$ incident to $u$. We claim that

$$
\operatorname{deg}_{G}(u) \leq 2 \quad \text { for every } u \in V(G) .
$$

Indeed, take $u v_{1} \in E(G)$; we can assume, without loss, that $u=R_{\pi / 2}\left(y, v_{1}\right)$. If $v_{3}=R_{\pi / 2}(y, u) \in P$, then we conclude that $x v_{3}>x y$ or $x v_{1}>x y$, because $\angle x y v_{3} \geq \pi / 2$ or $\angle x y v_{1} \geq \pi / 2$. This contradicts the fact that $x y$ is the diameter of $P$. Similarly, if $v_{2}$ and $v_{4}$ are defined as $u=R_{\pi / 2}\left(x, v_{4}\right)$ and $v_{2}=R_{\pi / 2}(x, u)$, then at most one of $v_{2}$ or $v_{4}$ can be in $P$.

Claim. All paths in $G$ have length at most 2.

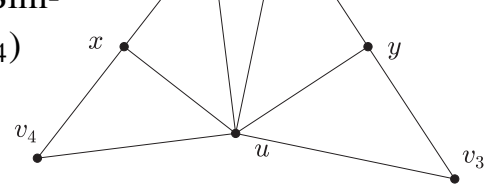

Proof. We prove this claim by contradiction. Suppose we can have a path of length 3 or more. To assist us, let us place our points on a cartesian coordinate system with our diameter $x y$ relabeled as the points $(0,0)$ and $(r, 0)$, furthermore, assume $p, q \geq 0$ and that the four vertices of the path of length 3 are $(p,-q),(q, p),(r-p, q-r)$, and $(r-q, r-p)$. Our aim is to show that the distance between $(r-q, r-p)$ and $(r-p, q-r)$ contradicts that $r$ is the diameter of $P$. Now, if paths of length 3 were possible, the distance between every pair of points in the figure on the right must be less than or equal to $r$. Since $d((p,-q),(q, p)) \leq r$, we have $p^{2}+q^{2} \leq r^{2} / 2$.

Now let us analyze the square of the distance from $(r-q, r-p)$ to $(r-p, q-r)$. Because $2\left(p^{2}+q^{2}\right) \geq(p+q)^{2}$, it follows that

$$
\begin{aligned}
d^{2}((r-q, r-p),(r-p, q-r)) & =(-q+p)^{2}+(2 r-p-q)^{2} \\
& =4 r^{2}-4 r(p+q)+2\left(p^{2}+q^{2}\right) \\
& \geq 4 r^{2}-4 \sqrt{2} r \sqrt{p^{2}+q^{2}}+2\left(p^{2}+q^{2}\right) \\
& =\left(2 r-\sqrt{2\left(p^{2}+q^{2}\right)}\right)^{2} .
\end{aligned}
$$

But $\sqrt{2\left(p^{2}+q^{2}\right)} \leq r$, so $\left(2 r-\sqrt{2\left(p^{2}+q^{2}\right)}\right) \geq r$ and thus

$$
d^{2}((r-q, r-p),(r-p, q-r)) \geq r^{2} .
$$

Equality occurs if and only if $p=r / 2$ and $q=r / 2$; otherwise, the distance between 
$(r-q, r-p)$ and $(r-p, q-r)$ is strictly greater than $r$, contradicting the fact that the diameter of $P$ is $r$. Therefore if $p \neq r / 2$ or $q \neq r / 2$ then there is no path of length 3. In the case that $p=r / 2$ and $q=r / 2$ the points $(q, p)$ and $(r-q, r-p)$ become the same and so do the points $(p,-q)$ and $(r-p, q-r)$. Thus we are left with a path of length 1 .

It follows from (10) and the Claim that all paths of length 2 are disjoint. Thus, $G$ is the union of disjoint paths of length at most 2. If $a$ denotes the number of paths of length 2 and $b$ the number of paths of length 1 , then

$$
|E(G)|=2 a+b \quad \text { and } \quad 3 a+2 b \leq n-2 .
$$

Recall from (9) that either

$$
\operatorname{deg}_{\pi / 2}(x)+\operatorname{deg}_{\pi / 2}(y)=|E(G)| \quad \text { or } \quad \operatorname{deg}_{\pi / 2}(x)+\operatorname{deg}_{\pi / 2}(y)=|E(G)|+1 .
$$

If $\operatorname{deg}_{\pi / 2}(x)+\operatorname{deg}_{\pi / 2}(y)=|E(G)|$, then

$$
2|E(G)|=4 a+2 b \leq n-2+a \leq n-2+\frac{n-2}{3},
$$

so $\operatorname{deg}_{\pi / 2}(x)+\operatorname{deg}_{\pi / 2}(y)=|E(G)| \leq \frac{2}{3}(n-2)$. Moreover, if

$$
\operatorname{deg}_{\pi / 2}(x)+\operatorname{deg}_{\pi / 2}(y)=|E(G)|+1,
$$

then $b \geq 1$ and we get a minor improvement,

$$
2|E(G)|=4 a+2 b \leq n-2+a \leq n-4+\frac{n-2}{3},
$$

so $\operatorname{deg}_{\pi / 2}(x)+\operatorname{deg}_{\pi / 2}(y)=|E(G)|+1 \leq(2 n-7) / 3<\frac{2}{3}(n-2)$.

We are now ready to put everything together. Between the two points $x$ and $y$, we derived the bounds:

$$
\begin{aligned}
& \operatorname{deg}_{\pi / 2}(x)+\operatorname{deg}_{\pi / 2}(y) \leq \frac{2}{3}(n-2), \\
& \operatorname{deg}_{\pi / 4}^{+}(x)+\operatorname{deg}_{\pi / 4}^{+}(y) \leq(n-1), \\
& \operatorname{deg}_{\pi / 4}^{-}(x)+\operatorname{deg}_{\pi / 4}^{-}(y) \leq(n-1) .
\end{aligned}
$$

Because the degree of a point must take on an integer value, it must be the case that either $x$ or $y$ satisfies $\operatorname{deg}_{\pi / 2}+\operatorname{deg}_{\pi / 4}^{+}+\operatorname{deg}_{\pi / 4}^{-} \leq\lfloor(4 n-5) / 3\rfloor$.

\section{Small cases}

In this section we determine the exact values of $S_{\triangle}(n)$ when $3 \leq n \leq 9$.

Theorem 6. For $3 \leq n \leq 9, S_{\triangle}(3)=1, S_{\triangle}(4)=4, S_{\triangle}(5)=8, S_{\triangle}(6)=11$, $S_{\triangle}(7)=15, S_{\triangle}(8)=20$, and $S_{\triangle}(9)=28$. 


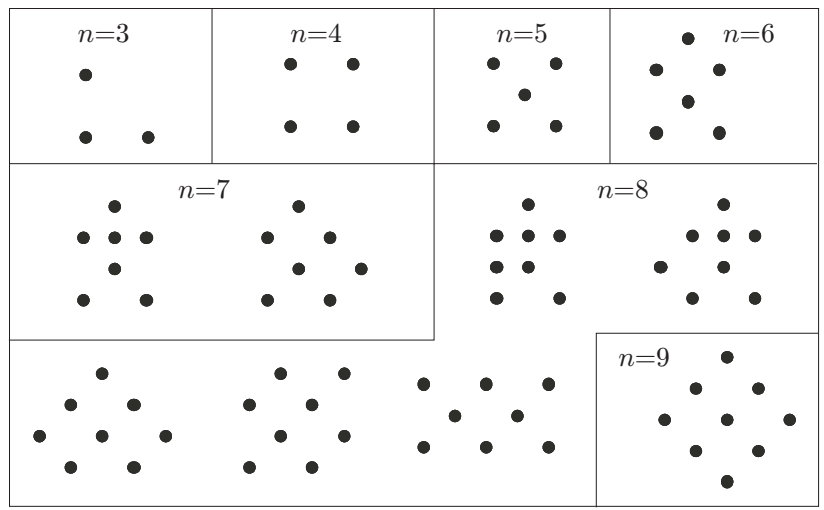

Figure 3. Optimal sets achieving equality for $S_{\triangle}(n)$.

The corresponding optimal sets are shown in Figure 3.

Proof. We begin with $n=3$. Since three points uniquely determine a triangle, and there is an IRT with three points, shown in Figure 4(a), this situation becomes trivial and we conclude that $S_{\triangle}(3)=1$.

Now let $n=4$. In Figure 4(b) we show a point set $P$ such that $S_{\triangle}(P)=4$. This implies that $S_{\triangle}(4) \geq 4$. However, $S_{\triangle}(4)$ is also bounded above by $\left(\begin{array}{l}4 \\ 3\end{array}\right)=4$. Hence, $S_{\triangle}(4)=4$.

To continue with the proof for the remaining values of $n$, we need the following two lemmas.

Lemma 7. Suppose $|P|=4$ and $S_{\triangle}(P) \geq 2$. The sets in parts (b)-(e) of Figure 4 are the only possibilities for such a set $P$, not counting symmetric repetitions.

Proof. Having $S_{\triangle}(P) \geq 2$ implies that we must always have more than one IRT in $P$. Hence, we can begin with a single IRT and examine the possible ways of adding a point and producing more IRTs. We accomplish this task in Figure 4(a). The 10 numbers in the figure indicate the location of a point, and the total number of IRTs after its addition to the set of black dots. All other locations not labeled with a number do not increase the number of IRTs. Therefore, except for symmetries, all the possibilities for $P$ are shown in Figure 4(b)-(e).

Lemma 8. Let $P$ be a finite set with $|P|=n$. Suppose that $S_{\triangle}(A) \leq b$ for all $A \subseteq P$ with $|A|=k$. Then

$$
S_{\triangle}(P) \leq\left\lfloor\frac{n(n-1)(n-2) b}{k(k-1)(k-2)}\right\rfloor .
$$

Proof. Suppose that within $P$, every $k$-point configuration contains at most $b$ IRTs. The number of IRTs in $P$ can then be counted by adding all the IRTs in every $k$ point subset of $P$. However, in doing so, we end up counting a fixed IRT exactly 

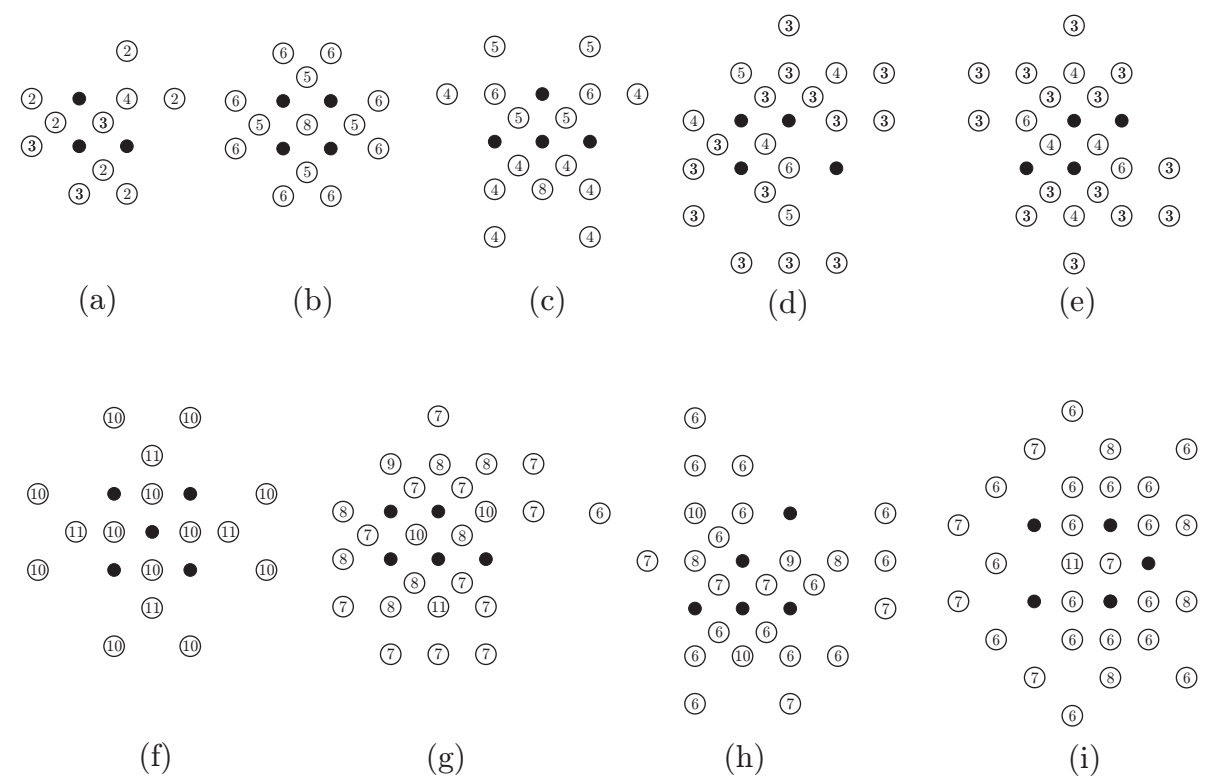

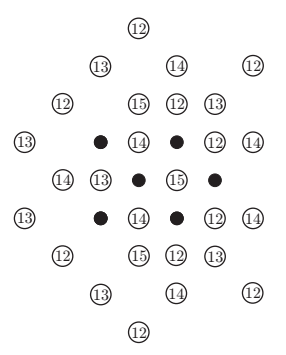

(j)

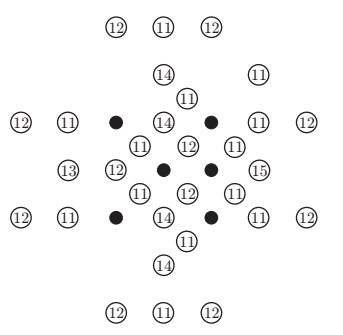

$(\mathrm{k})$

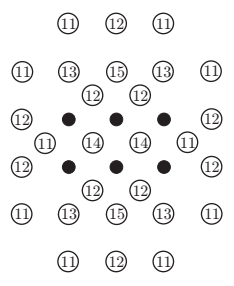

(1)

(11)

$$
\begin{aligned}
& \text { (13) (13) (12) (11) } \\
& \text { (1) (12) - (11) (11) (11) } \\
& \text { (12) } \bullet \text { (12) } \bullet \text { (11) } \\
& \text { (14) (II) (11) } \\
& \text { (11) (13) (1) (11) } \\
& \text { (11) (11) (111) } \\
& \text { (m) }
\end{aligned}
$$

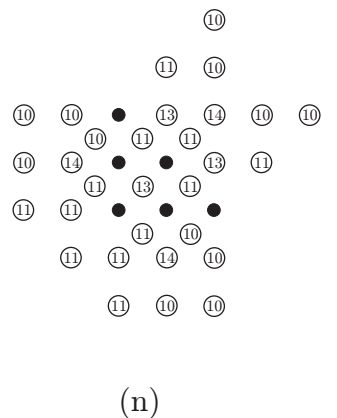

Figure 4. Proof of Theorem 6. Each circle with a number indicates the location of a point and the total number of IRTs resulting from its addition to the base set of black dots. 
$\left(\begin{array}{l}n-3 \\ k-3\end{array}\right)$ times. Because $S_{\triangle}(A) \leq b$ we get,

$$
\left(\begin{array}{l}
n-3 \\
k-3
\end{array}\right) S_{\triangle}(P)=\sum_{\substack{A \subseteq P \\
|A|=k}} S_{\triangle}(A) \leq\left(\begin{array}{l}
n \\
k
\end{array}\right) b .
$$

Notice that $S_{\triangle}(P)$ can only take on integer values so,

$$
S_{\triangle}(P) \leq\left\lfloor\frac{\left(\begin{array}{l}
n \\
k
\end{array}\right) b}{\left(\begin{array}{l}
n-3 \\
k-3
\end{array}\right)}\right\rfloor=\left\lfloor\frac{n(n-1)(n-2) b}{k(k-1)(k-2)}\right\rfloor .
$$

Now suppose $|P|=5$. If $S_{\triangle}(A) \leq 1$ for all $A \subseteq P$ with $|A|=4$, then by Lemma 8 , $S_{\triangle}(P) \leq 2$. Otherwise, by Lemma $7, P$ must contain one of the four sets shown in Figure 4(b)-(e). The result now follows by examining the possibilities for producing more IRTs by placing a fifth point in the four distinct sets. In Figure 4(b)-(e), we accomplish this task. Just as in Lemma 7, every number in a figure indicates the location of a point, and the total number of IRTs after its addition to the set of black dots. It follows that the maximum value achieved by placing a fifth point is 8 and so $S_{\triangle}(5)=8$. The point set that uniquely achieves equality is shown in Figure 4(f). Moreover, there is exactly one set $P$ with $S_{\triangle}(P)=6$ (shown in Figure 4(g)), and two sets $P$ with $S_{\triangle}(P)=5$ (Figures 4(h) and 4(i)).

Now suppose $|P|=6$. If $S_{\triangle}(A) \leq 4$ for all $A \subseteq P$ with $|A|=5$, then by Lemma 8, $S_{\triangle}(P) \leq 8$. Otherwise, $P$ must contain one of the sets in Figure 4(f)-(i). We now check all possibilities for adding more IRTs by joining a sixth point to our four distinct sets. This is shown in Figure 4(f)-(i). It follows that the maximum value achieved is 11 and so $S_{\triangle}(6)=11$. The point set that uniquely achieves equality is shown in Figure 4(j). Also, except for symmetries, there are exactly three sets $P$ with $S_{\triangle}(P)=10$ (Figure 4(k)-(m)) and only one set $P$ with $S_{\triangle}(P)=9$ (Figure 4(n)).

Now suppose $|P|=7$. If $S_{\triangle}(A) \leq 8$ for all $A \subseteq P$ with $|A|=6$, we have $S_{\triangle}(P) \leq 14$, by Lemma 8 . Otherwise, $P$ must contain one of the sets in parts (j)-(n) of Figure 4. We now check all possibilities for adding more IRTs by joining a seventh point to our 5 distinct configurations. We complete this task in parts (j)-(n). Because the maximum value achieved is 15 , we deduce that $S_{\triangle}(7)=15$. In this case, there are exactly two point sets that achieve 15 IRTs.

The proof for the values $n=8$ and $n=9$ follows along the same lines, but there are many more intermediate sets to be considered. We omit the details.

Inspired by our method used for proving exact values of $S_{\triangle}(n)$, a computer algorithm was devised to construct the best one-point extension of a given base set. This algorithm, together with appropriate heuristic choices for some initial sets, led to the construction of point sets with many IRTs giving us our best lower 


\begin{tabular}{|c|cccccccccccccccc|}
\hline$n$ & 10 & 11 & 12 & 13 & 14 & 15 & 16 & 17 & 18 & 19 & 20 & 21 & 22 & 23 & 24 & 25 \\
\hline bound & 35 & 43 & 52 & 64 & 74 & 85 & 97 & 112 & 124 & 139 & 156 & 176 & 192 & 210 & 229 & 252 \\
\hline
\end{tabular}

Table 1. Best lower bounds for $S_{\triangle}(n)$.

bounds for $S_{\triangle}(n)$ when $10 \leq n \leq 25$. These lower bounds are shown in Table 1 and the point sets achieving them in Figure 5.

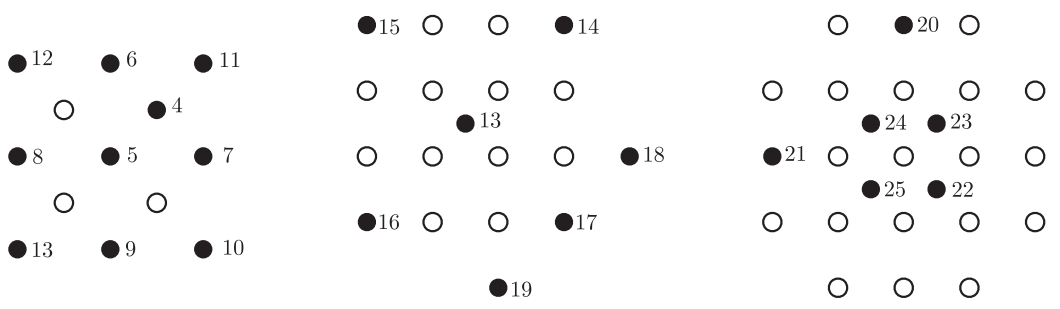

Figure 5. Best constructions $A_{n}$ for $n \leq 25$. Each set $A_{n}$ is obtained as the union of the starting set (in white) and the points with label $\leq n$. The value $S_{\triangle}\left(A_{n}\right)$ is given by Table 1 .

\section{Acknowledgements}

We thank Virgilio Cerna who, as part of the CURM mini-grant that supported this project, helped to implement the program that found the best lower bounds for smaller values of $n$. We also thank an anonymous referee for some useful suggestions and improvements to the presentation.

\section{References}

[Ábrego and Fernández-Merchant 2000] B. M. Ábrego and S. Fernández-Merchant, "On the maximum number of equilateral triangles. I", Discrete Comput. Geom. 23:1 (2000), 129-135. MR 2000i:05006 Zbl 0963.52007

[Brass 2002] P. Brass, “Combinatorial geometry problems in pattern recognition”, Discrete Comput. Geom. 28:4 (2002), 495-510. MR 2003m:68114 Zbl 1011.68117

[Brass and Pach 2005] P. Brass and J. Pach, "Problems and results on geometric patterns", pp. 17-36 in Graph theory and combinatorial optimization, edited by D. Avis et al., GERAD 25th Anniv. Ser. 8, Springer, New York, 2005. MR 2006f:05051 Zbl 1083.68110

[Brass et al. 2005] P. Brass, W. Moser, and J. Pach, Research problems in discrete geometry, Springer, New York, 2005. MR 2006i:52001 Zbl 1086.52001

[Elekes and Erdős 1994] G. Elekes and P. Erdős, "Similar configurations and pseudo grids", pp. 85104 in Intuitive geometry (Szeged, 1991), edited by G. F. Tâoth and K. Bèorèoczky, Colloq. Math. Soc. János Bolyai 63, North-Holland, Amsterdam, 1994. MR 97b:52020 
[Erdős and Purdy 1971] P. Erdős and G. Purdy, "Some extremal problems in geometry", J. Combinatorial Theory Ser. A 10 (1971), 246-252. MR 43 \#1045 Zbl 0219.05006

[Erdős and Purdy 1975] P. Erdős and G. Purdy, "Some extremal problems in geometry, III", Congressus Numerantium 14 (1975), 291-308. MR 52 \#13650 Zbl 0328.05018

[Erdős and Purdy 1976] P. Erdős and G. Purdy, "Some extremal problems in geometry, IV", Congressus Numerantium 17 (1976), 307-322. MR 55 \#10292 Zbl 0345.52007

[Laczkovich and Ruzsa 1997] M. Laczkovich and I. Z. Ruzsa, "The number of homothetic subsets", pp. 294-302 in The mathematics of Paul Erdös, II, edited by R. Graham and J. Nesetril, Algorithms Combin. 14, Springer, Berlin, 1997. MR 98e:52017 Zbl 0871.52012

Received: 2010-01-14 Revised: 2011-02-27 Accepted: 2011-02-27

bernardo.abrego@csun.edu Department of Mathematics, California State University, 18111 Nordhoff Street, Northridge, CA 91330-8313, United States http://www.csun.edu/ ba70714

silvia.fernandez@csun.edu Department of Mathematics, California State University, 18111 Nordhoff Street, Northridge, CA 91330-8313, United States http://www.csun.edu/ sf70713

david.roberts.0@csun.edu Department of Mathematics, California State University, 18111 Nordhoff Street, Northridge, CA 91330-8313, United States 
pjm.math.berkeley.edu/involve

\section{EDITORS}

MANAGING EDITOR

Kenneth S. Berenhaut, Wake Forest University, USA， berenhks@wfu.edu

\section{BOARD OF EDITORS}

John V. Baxley

Arthur T. Benjamin

Martin Bohner

Nigel Boston

Amarjit S. Budhiraja

Pietro Cerone

Scott Chapman

Jem N. Corcoran

Michael Dorff

Sever S. Dragomir

Behrouz Emamizadeh

Errin W. Fulp

Andrew Granville

Jerrold Griggs

Ron Gould

Sat Gupta

Jim Haglund

Johnny Henderson

Natalia Hritonenko

Charles R. Johnson

Karen Kafadar

K. B. Kulasekera

Gerry Ladas

David Larson

Suzanne Lenhart
Wake Forest University, NC, USA baxley@wfu.edu

Harvey Mudd College, USA

benjamin@hmc.edu

Missouri U of Science and Technology, USA bohner@mst.edu

University of Wisconsin, USA

boston@math.wisc.edu

U of North Carolina, Chapel Hill, USA

budhiraj@email.unc.edu

Victoria University, Australia

pietro.cerone@vu.edu.au

Sam Houston State University, USA

scott.chapman@shsu.edu

University of Colorado, USA

corcoran@colorado.edu

Brigham Young University, USA

mdorff@math.byu.edu

Victoria University, Australia sever@matilda.vu.edu.au

The Petroleum Institute, UAE bemamizadeh@pi.ac.ae

Wake Forest University, USA fulp@wfu.edu

Université Montréal, Canada andrew@dms.umontreal.ca

University of South Carolina, USA griggs@math.sc.edu

Emory University, USA rg@mathcs.emory.edu

U of North Carolina, Greensboro, USA sngupta@uncg.edu

University of Pennsylvania, USA jhaglund@math.upenn.edu

Baylor University, USA johnny_henderson@baylor.edu

Prairie View A\&M University, USA nahritonenko@pvamu.edu

College of William and Mary, USA

crjohnso@math.wm.edu

University of Colorado, USA

karen.kafadar@cudenver.edu

Clemson University, USA

kk@ces.clemson.edu

University of Rhode Island, USA

gladas@math.uri.edu

Texas A\&M University, USA

larson@math.tamu.edu

University of Tennessee, USA

lenhart@math.utk.edu
Chi-Kwong Li

Robert B. Lund

Gaven J. Martin

Mary Meyer

Emil Minchev

Frank Morgan

Mohammad Sal Moslehian

Zuhair Nashed

Ken Ono

Joseph O'Rourke

Yuval Peres

Y.-F. S. Pétermann

Robert J. Plemmons

Carl B. Pomerance

Bjorn Poonen

James Propp

Józeph H. Przytycki

Richard Rebarber

Robert W. Robinson

Filip Saidak

Andrew J. Sterge

Ann Trenk

Ravi Vakil

Ram U. Verma

John C. Wierman
College of William and Mary, USA ckli@math.wm.edu

Clemson University, USA lund@clemson.edu

Massey University, New Zealand g.j.martin@massey.ac.nz

Colorado State University, USA meyer@stat.colostate.edu

Ruse, Bulgaria

eminchev@hotmail.com

Williams College, USA

frank.morgan@williams.edu

Ferdowsi University of Mashhad, Iran moslehian@ferdowsi.um.ac.ir

University of Central Florida, USA

znashed@mail.ucf.edu

University of Wisconsin, USA

ono@math.wisc.edu

Smith College, USA

orourke@cs.smith.edu

Microsoft Research, USA peres@microsoft.com

Université de Genève, Switzerland petermann@math.unige.ch

Wake Forest University, USA plemmons@wfu.edu

Dartmouth College, USA carl.pomerance@dartmouth.edu

UC Berkeley, USA poonen@math.berkeley.edu

U Mass Lowell, USA jpropp@cs.uml.edu

George Washington University, USA przytyck@gwu.edu

University of Nebraska, USA rrebarbe@math.unl.edu

University of Georgia, USA rwr@cs.uga.edu

U of North Carolina, Greensboro, USA

f_saidak@uncg.edu

Honorary Editor

andy@ajsterge.com

Wellesley College, USA

atrenk@wellesley.edu

Stanford University, USA

vakil@math.stanford.edu

University of Toledo, USA

verma99@msn.com

Johns Hopkins University, USA

wierman@jhu.edu

\section{PRODUCTION}

Silvio Levy, Scientific Editor

Sheila Newbery, Senior Production Editor

Cover design: @2008 Alex Scorpan

See inside back cover or http://pjm.math.berkeley.edu/involve for submission instructions.

The subscription price for 2011 is US \$100/year for the electronic version, and \$130/year (+\$35 shipping outside the US) for print and electronic. Subscriptions, requests for back issues from the last three years and changes of subscribers address should be sent to Mathematical Sciences Publishers, Department of Mathematics, University of California, Berkeley, CA 94704-3840, USA.

Involve (ISSN 1944-4184 electronic, 1944-4176 printed) at Mathematical Sciences Publishers, Department of Mathematics, University of California, Berkeley, CA $94720-3840$ is published continuously online. Periodical rate postage paid at Berkeley, CA 94704, and additional mailing offices.

Involve peer review and production are managed by EditFLOW ${ }^{\mathrm{TM}}$ from Mathematical Sciences Publishers.

PUBLISHED BY

mathematical sciences publishers

http://msp.org/

A NON-PROFIT CORPORATION

Typeset in LATEX

Copyright $@ 2011$ by Mathematical Sciences Publishers 


\section{involve 2011 vol. $4 \quad$ no. 1}

The arithmetic of trees

ADRIANO BRUNO AND DAN YASAKI

Vertical transmission in epidemic models of sexually transmitted diseases with isolation from reproduction

Daniel Maxin, Timothy Olson and Adam Shull

On the maximum number of isosceles right triangles in a finite point set

Bernardo M. Ábrego, Silvia Fernández-Merchant and David B.

ROBERTS

Stability properties of a predictor-corrector implementation of an implicit linear

multistep method

SCOTT SARRA AND Clyde MEAdor

Five-point zero-divisor graphs determined by equivalence classes

FLORIDA LEVIDIOTIS AND SANDRA SPIROFF

A note on moments in finite von Neumann algebras

Jon BANNON, Donald HAdWIn AND MAUREEN JEFFERY

Combinatorial proofs of Zeckendorf representations of Fibonacci and Lucas products Duncan McGregor and Michael Jason Rowell

A generalization of even and odd functions

Micki BALAICH AND MATtheW ONDRUS 